\title{
Formation of Accelerated Particle Beam in Isochronous Cyclotron
}

\author{
A.M. VORONIN ${ }^{a}$, J. PARTYKA ${ }^{b}$ AND A. AldiBEKOVA ${ }^{c, *}$ \\ ${ }^{a}$ Almaty Technological University, 100, Tole Bi Str., 050012 Almaty, Kazakhstan \\ ${ }^{b}$ Lublin University of Technology, Nadbystrzycka 38a, 20-618 Lublin, Poland \\ ${ }^{c}$ K.I. Satpayev Kazakh National Technical University, 22, Satpayev Str., 050013 Almaty, Kazakhstan

\begin{abstract}
In the paper there are given calculated and experimental data which allow to form necessary beam parameters for a precise experiment in nuclear physics. An intensity of a beam extracted from an isochronous cyclotron acceleration chamber $\left(E_{\mathrm{p}}=30 \mathrm{MeV}\right)$ can reach $15 \mu \mathrm{A}$ with an energy spread of $3 \times 10^{-3}$. Accelerated ion beams of such kind are widely applicable in nuclear physical experiments.
\end{abstract}

DOI: $10.12693 /$ APhysPolA.125.1408

PACS: 29.27.Bd, 29.20.dg, 41.85.-p

\section{Introduction}

In the literature there is usually considered a dependence of the one of main beam parameters - the value of energy spread, $\Delta E / E$, on cyclotron characteristics for magnetic field by form closed to isochronous one [1]. Since the output of ion beams from considered installation is realized in decreasing field, there was carried out an estimation of energetic spread of the beam with finite phase width $\Delta \varphi$ in case of one-revolution output for real magnetic field that has a decreasing area.

\section{Experiment on formation of precise beam of accelerated ions in cyclotron}

The expression for high-frequency phase of ion in the function of the revolution number was added by the term, taking into account the exponential increase of the phase at the end of acceleration

$$
\varphi_{(n)}=\varphi_{0}+\Delta \varphi+\psi\left(2 \pi \frac{n}{N}+\gamma\right)+\varepsilon_{n}+\alpha l^{\beta n}
$$

where $\varphi, \gamma$ are amplitude and phase of the change of $\varphi_{(n)}$ with the period $N ; \varepsilon=\mathrm{d} \varphi / \mathrm{d} n=2 \pi(\mathrm{d} f / f)=$ $-2 \pi(\mathrm{d} B / B)$ is the change of the phase, stipulated by the change of the frequency of the accelerating voltage or the electric intensity. In the considered case the finite decrease of the field gives additional terms into the expression of the relative deviation of the ion energy

$$
\begin{aligned}
\frac{\sigma E}{E} & =\frac{\alpha l^{\beta n}}{\beta n}\left\{\tan \varphi_{0}-\Delta \varphi+\frac{\varepsilon}{\beta}-\varepsilon_{n}-\frac{1}{4} \alpha l^{\beta n}\right. \\
+ & \frac{\beta \psi}{\beta^{2}+(2 \pi / N)^{2}}\left[\frac{2 \pi}{N} \cos \left(\frac{2 \pi}{N} n+\gamma\right)\right. \\
& \left.\left.-\beta \sin \left(\frac{2 \pi}{N} n+\gamma\right)\right]\right\} .
\end{aligned}
$$

As it follows from the assumption carried out, for concrete conditions of the beam acceleration, the finite value

\footnotetext{
*corresponding author; e-mail: aitkul_86@mail.ru
}

of the energetic spread $\Delta E / E=4 \times 10^{-3}$ for phase band $\Delta \varphi=6^{\circ}$ at the instability of $\sigma U / U=3 \times 10^{-3}$. The analysis of the passage of resonance values of work frequencies in the process of acceleration showed that for considered installation (the Alma-Ata isochronous cyclotron, $\mathrm{U}-150 \mathrm{M})$ the most serious fact is the dynamical passage of the resonance at the first harmonic in the region of the acceleration radius, $R=58 \mathrm{~cm}$, and link resonance with $\nu_{\mathrm{r}}=2 \nu_{z}$, at $R=63 \mathrm{~cm}$. Computer estimations showed the possibilities of successful passage of these resonances by using the acceleration regime with total set of ions energies per one revolution $\left(U_{0} \geq 70 \mathrm{kV}\right)$ and by limiting the values of axial amplitudes of oscillations up to $a_{z} \leq 5 \mathrm{~mm}$. More precise determination of conditions of initial and following processes of ions accelerations was carried out on the basis of numerical calculations of beam motion dynamics, having finite time and spatial values of its emittances. The measurements of the emittance and the distribution of the phase density of the current, on the one hand, allows practically to perfect the optimal optics of the system "source-puller" and, on the other hand, the obtained information is the basis of both of computational and practical aspects of optimization of initial and following processes of current acceleration [2].

The preliminary obtaining of methodical information relatively of choice of places for the arrangement of forming collimators, testers, dimensions of detecting collimators and their slits, coordinated with the increment of orbit's radius and with section of measured beam, carried out accordingly with matrix relation of two azimuth states of beam's coordinates in one magnetic field. Final determinations of the form of emittance contour were carried out by the combination of results of numerical integration of equations of ions motions with data of measurements. Experiments were carried out on the small-sized cyclotron which was the full-scale model of the centre for the source of Penning's kind that is in regular operation on the main machine.

Registration of the internal beam current in the accelerator was realized with the use of 10 Faraday cups. They were collimated by a slotted diaphragm with $1 \mathrm{~mm}$ 
aperture. A block of 10 cups with a common collimator was set on a carriage of a mobile probe which was moved along the radius of the acceleration chamber between the dees. The measured current was applied via a switch to a computer that performed a slow time analysis. Values of the current were stored in the memory of the computer. Such a method allows to observe axial and radial emittances of an accelerated-ion beam in a cyclotron.

The distribution of current density for typical geometry is shown in Fig. 1a, and corresponding iso-densities of the current - in Fig. 1b. Dashes on the line $r_{0}^{\prime}$ (Fig. 1b) mark the one-degree change of the angle of ion emission relative to the central trajectory at constant HF-phase of the voltage. Measured dependences give values of $340 \mathrm{~mm}$ mrad and $164 \mathrm{~mm} \mathrm{mrad} \mathrm{for} 100 \%$ and $80 \%$ of lines of the density of radial emittance and, correspondingly, $560 \mathrm{~mm} \mathrm{mrad}$ and $190 \mathrm{~mm} \mathrm{mrad} \mathrm{in} \mathrm{axial} \mathrm{plane}$ $\left(U_{0}=65 \mathrm{kV}\right)$, besides the angular spread of the radial partial emittance at the output from the filler is $\pm 4 \%$ for $80 \%$ of density's lines.
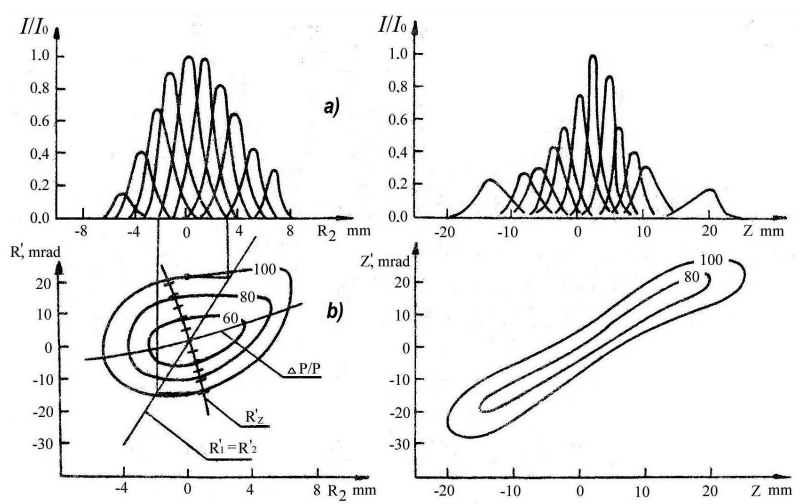

Fig. 1. Emittance of internal beam in radial and axial phase planes: (a) the distribution of current density for typical geometry, (b) iso-densities of the current.

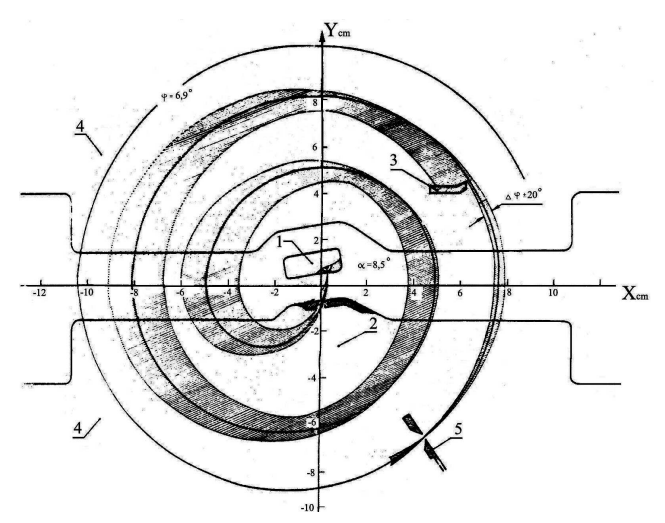

Fig. 2. Initial orbits of the beam in the central region of the cyclotron: 1 - the source, 2 - the filler, 3 - the post, 4 - the bundle, 5 - the angular collimator.

Knowledge of radial value of partial emittance of the beam allows to keep track of whole process of its transfor- mation during the acceleration, beginning from the first accelerating clearance and directly before the exit into the deflecting system. As numerical calculations show, in spite of some distortions of phase contour, radial dimensions of final beam, limited by initial $80 \%$ line of the density, are in limits of $2 A_{H}=2 \mathrm{~mm}$ at minimum division of blobs, $\Delta r=2 \mathrm{~mm}$. On the base of fulfilled investigations there is carried out the consideration of phase and angular selection of the beam with the purpose to separate its final orbits (Fig. 2).

\section{Extraction and time-structure formation of accelerated-ion beam in cyclotron}

Usage of different techniques of beam extraction is predestined by a variety of sorts of accelerated ions. A choice of extraction technique was determined by main requests which are usually demanded from beam extraction system: matching of output beam emittance with external transportation channel acceptance; maximum possible current output factor; preservation of beam quality while extracting.

To extract a beam of light ions $\left({ }^{2} \mathrm{H}^{+},{ }^{3} \mathrm{He}^{2+},{ }^{4} \mathrm{He}^{2+}\right)$ accelerated up to the maximum energy of $70 \mathrm{MeV}$, it was supposed to realize the usual technique with an electrostatic deflector, a magnetic current channel, an iron focusing shield, and a system focusing on the ion guide axis. Provision of high output efficiency of the system (on condition that deflector acceptance matches output beam emittance) is connected with an achievement of sufficient separation of the last orbits that is necessary for placement of a deflecting system septum. An increase in energy of accelerated particles with simultaneous decrease in energy gain per turn (due to single-dee design of the accelerating system) leads to appreciably less separation of the last orbits that is estimated as being less than $1 \mathrm{~mm}$. In this connection, a necessity arises for application of resonance technique of beam extraction using harmonic disturbances of magnetic field in the region of the last orbits of acceleration. As well for heavy ions, there are another techniques of accelerated ion beam extraction out of cyclic accelerators.

The main feature of the Alma-Ata cyclotron is the accelerating system, consisting of two $180^{\circ}$ dees. This system affords the large energy gain per turn thus decreasing influence of magnetic field disturbances and enlarging the turn separation at extraction radius. At the same time the necessity of placing the deflector inside the dee complicates substantially its design.

The high effective and reliable extraction system must meet the needs of necessary deflection and focusing of the beam and to be reliable under large heat load. At changing magnetic field in the wide range it is necessary also to take into account the changing of the shape of the orbit at extraction radius. For this reason a new electrostatic deflector with non-uniform electric field and with precise replacing of its parts without switching off high voltage potential was designed. This deflector located 
inside the dee is shown schematically in Fig. 3, the angular coordinates $\left(15^{\circ}, 55^{\circ}, 125^{\circ}\right)$ of three movable parts of the deflector are depicted. The fine displacement of these parts of the deflector may be made in the range of $\pm 7 \mathrm{~mm}$ with accuracy of $0.1 \mathrm{~mm}$ and be produced remotely from the main control panel with three pairs of hydraulic cylinders.

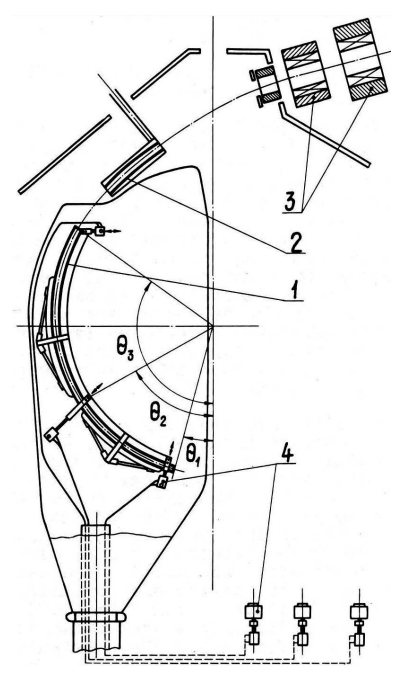

Fig. 3. Beam extraction systems: 1 - adjustable deflector, 2 - additional deflector, 3 - steering magnets, 4 - hydraulic cylinders.

This system is designed for increasing the resolution of a neutron spectrometer based on time-of-flight measurements by reducing the beam repetition rate. Using a selection slit, three successive accelerated particles bunches of every four ones are stopped at a collimator after sweeping off by high-voltage RF deflector. Only the bunches which see a zero resulting electric field between the electrodes of the deflector are able to pass the selection slit. Usually as a bunch selector a deflector with flat electrodes and sinusoidally varying potential difference between them is used.

An effective sweeping off system assumes absence of beam losses, minimum deflecting voltage, reliable design, simple tuning, and easy running. To meet these needs a deflection system was developed which is located in the fringing magnetic field of the cyclotron immediately after the main deflector. Such a system gives substantial reduction of deflection potential in comparison with ordinary one located in nonuniform magnetic field free space. The deflection system is shown in Fig. 3. An additional deflector (pulser) is located inside the accelerator chamber and outside the dee $\left(\theta=142-162^{\circ}\right)$, where the beam size is sufficiently small yet.

The deflector is composed of a pair of plates $30 \mathrm{~cm}$ long and separated by $3 \mathrm{~cm}$, in cross-section they have hyperbolic profile for increasing radial focusing of the beam. The results of the numerical calculations of the beam envelopes in the horizontal plane for different potentials at the deflector are shown in Fig. 4.
The experimentally measured beam emittance and ion transit time in the deflector have been taken into account. The minimum potential for $30 \mathrm{MeV}$ protons burst separation is $15 \mathrm{kV}$.

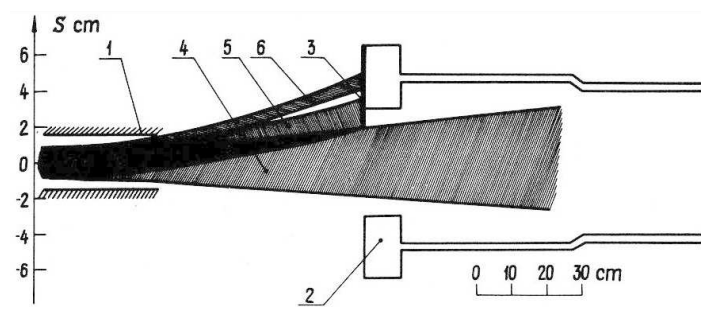

Fig. 4. Particles bursts sweeping off systems and beam envelopes: 1 - RF-selector, 2 - beamline, 3 - collimator, 4, 5, 6 - beams at potential difference of $0.15 \mathrm{kV}$ and $30 \mathrm{kV}$.

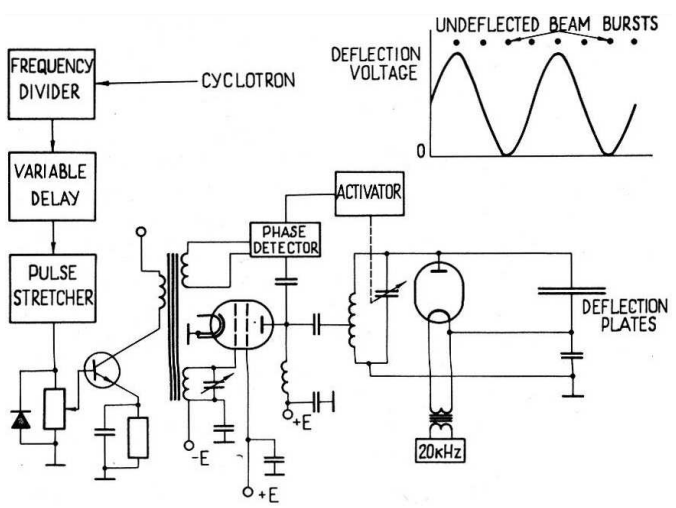

Fig. 5. Simplified scheme of particles bursts selection system.

A scheme of particles bursts selection system is shown in Fig. 5. The final section is a resonant $\mathrm{LC}$ circuit in which the selector is a capacitive element. In this circuit a sinusoidal voltage is excited. The frequency varies in the range of $2-4.5 \mathrm{MHz}$. The final amplifier stage consists of a power tetrode run in class AB with a total power of $0.8-1.0 \mathrm{~kW}$. The resulting potential difference generated between the electrodes of the selector varies sinusoidally from zero to the peak value. This signal is phased with the particles bunches, so that the interesting bunch passes through the deflector when the potential across the electrodes $V=0$ and $\mathrm{d} V / \mathrm{d} t=0$, too.

\section{Conclusions}

Calculations, carried out taking into account links of longitudinal and radial-cross kinds of motion, allowed to determine necessary conditions of initial collimation of the beam, allowing to limit both the angular spread of the beam and the phase width of blobs, separated at the azimuth of the deflector. The optimality of the geometry of the centre was checked by the consideration of the process of the acceleration in the axial plane taking into 
account links of radial and longitudinal kinds of the motion, besides there were determined concrete conditions of the axial collimation of the beam. For found cross- and longitudinal acceptances of the cyclotron there are determined computational final transverse emittances of the beam, $10^{-12} \mathrm{~mm} \mathrm{mrad}$, and value of its energy spread, $\Delta E / E=3 \times 10^{-3}$. Estimations of an intensity of formed beam, carried out taking into account of measured initial distribution of phase current density and of an influence of Coulomb's effect and the spatial charge, show that the maximum value of the average current at the final radius is $\approx 15 \mu \mathrm{A}$ for protons, accelerated up to the energy of $30 \mathrm{MeV}$.

The use of correctly-formed ions beam inside accelerating chamber of the cyclotron [3-5] allows to carry out the wide class of nuclear-physical investigations at the contemporary level.

\section{References}

[1] W.G. Davies, in: Cyclotrons and Their Applications, Proc. 13th Int. Conf., Vancouver 1992, Eds.: G. Dutto, M.K. Craddock, World Scientific Publishing, Singapore 1993, p. 372.

[2] W.J.G.M. Kleeven, J.L. Delhez, J.I.M. Botman, G.A. Webers, C.J. Timmermans, H.L. Hagedoorn, in Ref. [1], p. 384.

[3] A.M. Voronin, Vestnik Natsionalnoi Akademii Nauk Respubliki Kazakhstan 2, 19 (2012) (in Russian).

[4] A.M. Voronin, Poisk. Seriya yestestvennykh $i$ tekhnicheskikh nauk 2, 105 (2012) (in Russian).

[5] A.M. Voronin, in: Days of Science 2012, Proc. 8th Int. Sci. Pract. Conf., Praha 2012, Ed. Z. Cernák, Education and Science, Praha 2012, p. 27 (in Russian). 